\title{
Integrated Microsystems for Smart Applications
}

\author{
Thomas Otto, ${ }^{1,2 *}$ Steffen Kurth, ${ }^{1}$ Sven Voigt, ${ }^{1}$ Andreas Morschhauser, ${ }^{1}$ \\ Marco Meinig, ${ }^{1}$ Karla Hiller, ${ }^{1,2}$ Martin Moebius, ${ }^{2}$ and Martina Vogel ${ }^{1}$ \\ ${ }^{1}$ Fraunhofer Institute for Electronic Nano Systems ENAS, Technologie-Campus 3, 09126 Chemnitz, Germany \\ ${ }^{2}$ Center for Microtechnologies at Chemnitz University of Technology, 09107 Chemnitz, Germany
}

(Received November 4, 2017; accepted January 18, 2018)

Keywords: smart integrated system, smart health, smart power, smart mobility

More and more smart components and smart integrated systems enter into diverse innovative products and applications. They are key components and key technologies for industry 4.0 as well as Internet of Things and Services. The application areas include not only smartphones with advanced functionalities but also monitoring systems for sustainable production, smart agriculture, smart grid, and smart home as well as support systems for senior citizens to guarantee their more independent lifestyle. In this paper we describe different prototype developments. The main focus is on the systems of Fraunhofer Institute for Electronic Nano Systems ENAS (Fraunhofer ENAS) and the Center for Microtechnologies (ZfM) at Chemnitz University of Technology. The application fields of the prototypes are smart health, smart energy, and smart production.

\section{Introduction}

The particular strength of Fraunhofer ENAS lies in the development of smart systems for various applications. These smart systems combine electronic components with nano- and microsensors as well as actuators, communication units and self-sufficient power supply. Furthermore, smart systems can respond to each other, identify one another, and work in concert. Hence, they form the base for the Internet of Things. Smart systems are developed by using key enabling technologies and by integrating the knowledge from various disciplines. They benefit from the progress in nanoelectronics and nanotechnologies, but also from design methods and tool development.

It is clear that smart systems have steadily evolved in recent decades - and have developed even further-in their variety of technologies, materials, used physical effects, and application fields. With their offered variety of solution options, they are adaptable to many different application fields as well, especially at the moment and even more in the future pending requirements from the global challenges, mainly climate change, a secure energy supply, sustainable transport, sustainable production, demographic changes, and securing of health and well-being. These capabilities of smart systems thus result in extremely high expectations; they

*Corresponding author: e-mail: Thomas.Otto@enas.fraunhofer.de http://dx.doi.org/10.18494/SAM.2018.1797 
are ultimately a key technology. These same claims generate tremendous development pressure, by which the development will be further promoted and accelerated. ${ }^{(1)}$

What separates a smart system from a system that is purely reactive is the knowledge base, which ranges from a set of parameters for a feedback loop to embedded databases and algorithms. It is a necessary condition for the smartness of a system to provide safe and reliable autonomous operation under all relevant circumstances. The building blocks of a smart system are shown in Fig. 1. ${ }^{(2)}$

In this work, different examples and prototypes will be described in more detail. In the next sections we show different application fields.

\section{Smart Systems for Health}

\subsection{Point-of-care and point-of-need systems}

Decentralized chemical or biochemical testing represents another possible domain for smart systems. For such systems multiple terms exist including point-of-care testing, home testing, bedside testing, and point-of-need testing. All these terms describe a testing of chemical or biochemical parameters at or near the place the sample is acquired. The possible fields of application are huge: human and veterinary diagnostics, food safety and quality, environmental parameters, and many more. The motivation for the quick tests are as numerous as the fields of application: On the one hand, decentralized testing promises faster results (minutes to hours) and thus, for example, faster therapeutic decisions, which are crucial in emergency care or severe conditions such as sepsis. On the other hand, decentralized testing does not rely on laboratory infrastructures, which might just not be available (developing countries) or impaired (catastrophe settings). A third motivation results from the high grade of automation: Most of the tests are designed as sample-in-answer-out systems. Thus, they can be used even by untrained personnel or the patients themselves.

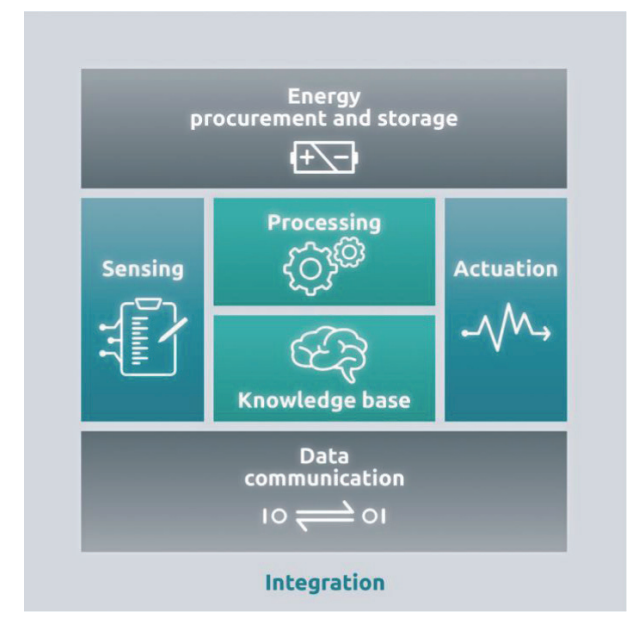

Fig. 1. (Color online) Building blocks of a smart system. ${ }^{(2)}$ 
For such decentralized testing systems, routine laboratory processes need to be miniaturized and automated. The scope of necessary functions includes liquid manipulation (pumps, valves, metering, ...), heating/cooling, reagent storage, sensing, communication, and power supply. While microfluidics is the key enabling technology for integrated liquid handling, heterogeneous technologies need to be combined to achieve compact and fully automated systems.

Three examples of smart systems for point-of-care applications are described below in more detail with respect to smart system aspects.

Digital pregnancy tests are a further development of urine test strips detecting human chorionic gonadotropin (hCG) in urine, which were introduced in $1976 .{ }^{(3)}$ The uncertainty of the user in the interpretation of detection lines was removed by the integration of technology. The digital test incorporates among the test strip itself also an optical sensor, readout electronics, a display, and a battery. Usually, these tests activate themselves, when the sample pad comes into contact with a liquid. With this wake-up feature, a long shelf life is reached. The disposable systems are optimized for user comfort and low cost.

Another example of smart systems for home testing are continuous glucose monitoring systems. ${ }^{(4)}$ These systems are attached to the patients body in the form of small patches and regularly (each 1 to $5 \mathrm{~min}$ ) measure the tissue glucose level for a time span of 5 to 14 days. The systems include glucose sensors, readout electronics, a battery, and a communication unit. Some of the systems are also integrated with an insulin pump and thus can directly regulate the blood sugar level of the patient.

Infectious disease testing is another important example for microfluidic smart systems. While lateral flow strips are capable of testing a limited number of biochemical parameters, more complex biomolecular sensing principles [microarrays, polymerase chain reaction (PCR), single nucleotide polymorphism (SNP)] require the integration of complex analysis procedures. In the European-Brazilian cooperation project PodiTrodi, a detection system for Chagas, a protozoan parasite, was developed. Figure 2 shows the developed microfluidic cartridge and the corresponding readout system. The test system uses a DNA-based real-time-PCR for the direct detection of the parasite DNA and an antibody-based test strip for the longterm monitoring of

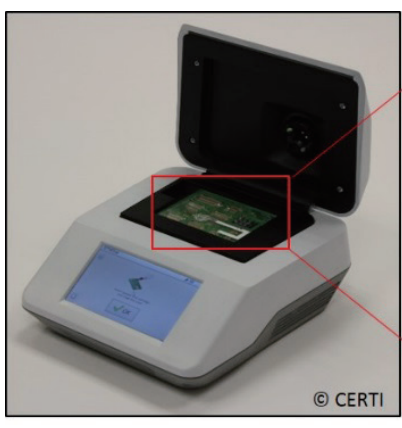

(a)

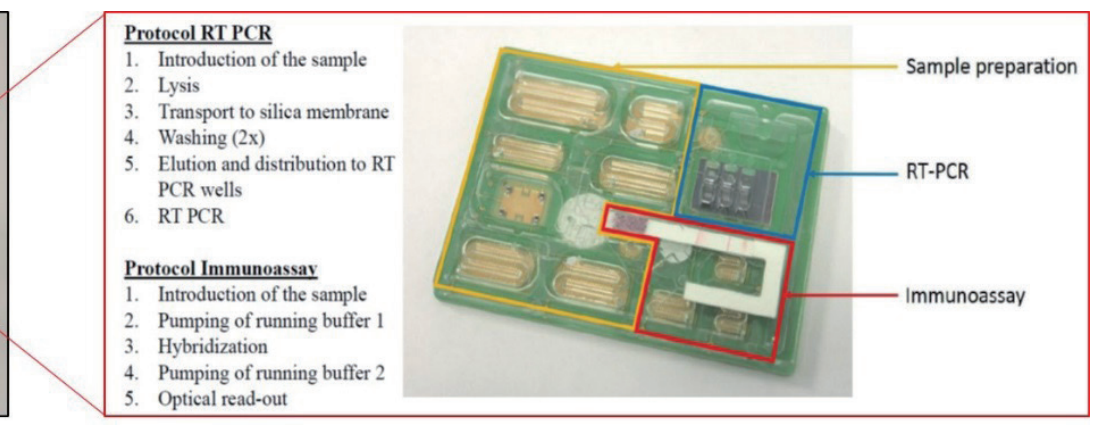

(b)

Fig. 2. (Color online) Microfluidic point-of-care system for Chagas detection composed of a readout unit (a) and a microfluidic cartridge (b). 
the immune response. The cartridge automizes a $2 \mathrm{~h}$ labor-intensive laboratory test into a 30 min sample-in-answer-out system, which can be performed by untrained medical staff.

These three examples show the huge potential of smart systems for point-of-care/point-ofneed applications. Further development will be driven by the integration of new measurement principles and more and more complex analysis procedures.

\subsection{Fabry-Pérot interferometer (FPI)}

Infrared spectroscopy is a powerful analysis method. Many substances can be realibly distinguished by their unique absorption spectra. Conventional analysis systems and spectrometers are complex and expensive instruments with very limited portability. The market requests small, fast, and robust analyzers for volatile organic compounds. Therefore, tunable FPIs and the development of tiny, robust, and fast spectrometers have been the focus of scientific research for several years. ${ }^{(5-7)}$ The authors already presented their work on tunable FPIs for infrared spectroscopy in Refs. 5, 8, and 9. The FPIs can be reliably and reproducibly fabricated with a high yield in MEMS wafer-level batch processes. Major improvements of the new FPI in comparison with previous designs are a significantly smaller bandwidth, a lower tuning voltage, a reduction of the chip size to $7 \times 7 \mathrm{~mm}^{2}$, and a large aperture of $2 \times 2 \mathrm{~mm}^{2}$.

The FPI consists of two semitransparent reflectors with high reflectivity (Fig. 3). The reflectors are aligned parallel to each other very precisely and build an optical resonator in between. The transmission spectrum of the resonator shows multiple narrow peaks and strong blocking of the incident waves in between. A transmittance maximum occurs for wavelengths that fulfill the interference condition of the resonator. By varying the distance between the reflectors, the wavelength at which the interference maximum occurs can be changed. Therefore, FPIs are ideally suited for use as tunable band-pass filters in optical applications.

The spectral transmittance was measured with a Fourier tramsform infrared spectrometer

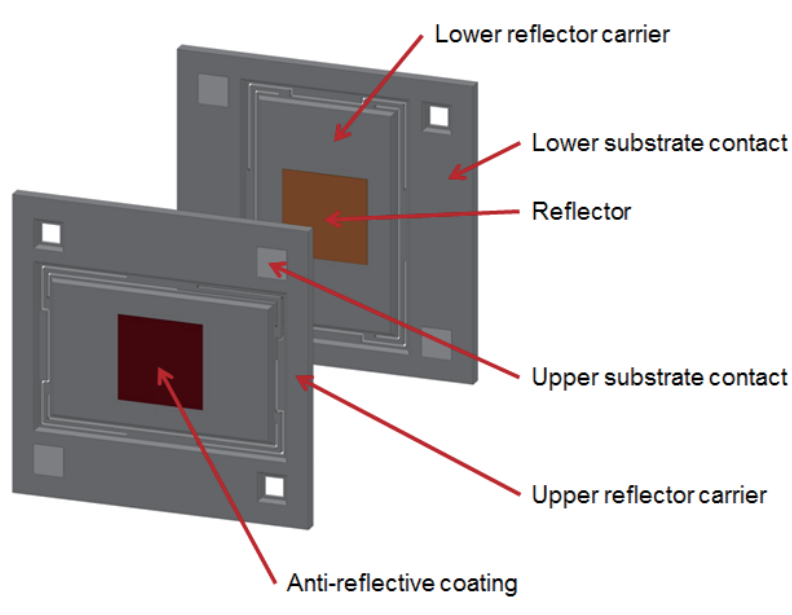

(a)

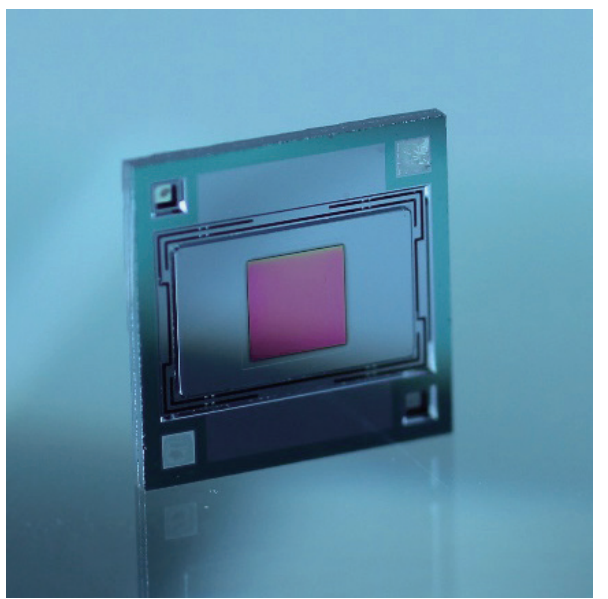

(b)

Fig. 3. (Color online) (a) 3D model of the FPI (exploded view) and (b) photograph of a fabricated FPI. 
(FTIR) (Bruker Vertex 70) with a spectral resolution of $4 \mathrm{~cm}^{-1}$. Figure 4(a) shows the tuning of the transmittance maxima for the 2nd and 3rd interference orders. The spectral range from 3.1 to $4.4 \mu \mathrm{m}$ can be used without the overlapping of modes, if an additional wide band-pass filter is used to block wavelengths outside of this range. For the 2nd interference order, the peak transmittance is between 81 and $91 \%$ and the full width at half maximum (FWHM) is between 36 and $70 \mathrm{~nm}$. The 2 nd interference order can be tuned from 5.5 to $4 \mu \mathrm{m}$. In the $3 \mathrm{rd}$ interference order, the peak transmittance is between 76 and $86 \%$ and the FWHM is between 25 and $28 \mathrm{~nm}$. The $3 \mathrm{rd}$ interference order can be tuned from 4 to $2.8 \mu \mathrm{m}$. For both orders, the tuning voltage is lower than $32 \mathrm{~V}$.

Applications for tunable FPIs are infrared measurements, spectral gas analysis, and spectral imaging. InfraTec $\mathrm{GmbH}$, for example, integrates the FPIs in tiny and robust microspectrometer modules. ${ }^{(8)}$ A schematic drawing of a complete set-up including an infrared emitter and a sample cell is shown in Fig. 4(b).

The spectrometer setup consists of an infrared emitter, a sample cell, an aperture window (IR band-pass filter), and a broadband pyroelectric detector. Pyroelectric detectors have a specific detectivity $\mathrm{D}^{*}$ of $5 \times 10^{8} \mathrm{~cm} \sqrt{\mathrm{Hz}} / \mathrm{W}$ and a flat frequency response up to approximately $70 \mathrm{~Hz}$. The complete spectrometer module fits in a TO-8 housing.

\section{Smart Systems for Power}

The growing share of renewable energy sources, their high fluctuations and the everchanging environmental conditions such as high summer temperatures or ice loads are major challenges for electric power transmission system operators and their transmission grid. The determination of the prevailing operational and environmental conditions along the overhead line by a smart sensor system allows an increase in the operational reliability. In close collaboration, ZfM, Fraunhofer ENAS, Fraunhofer IZM, First Sensor Microelectronic

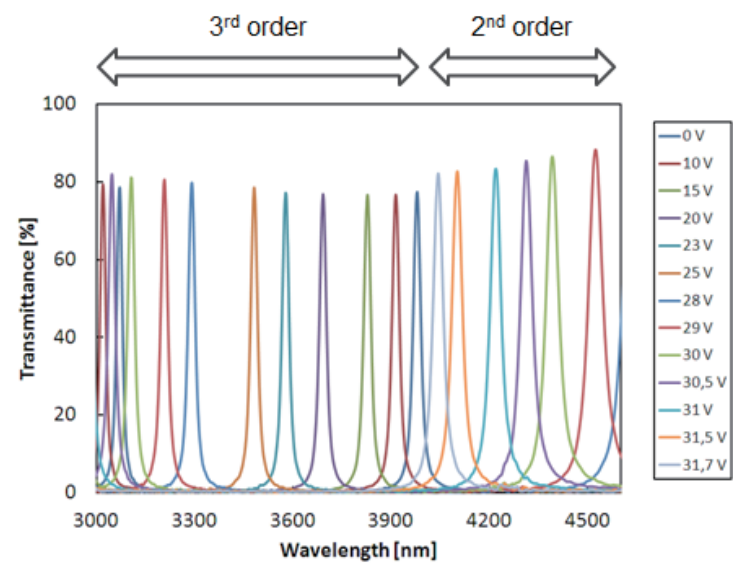

(a)

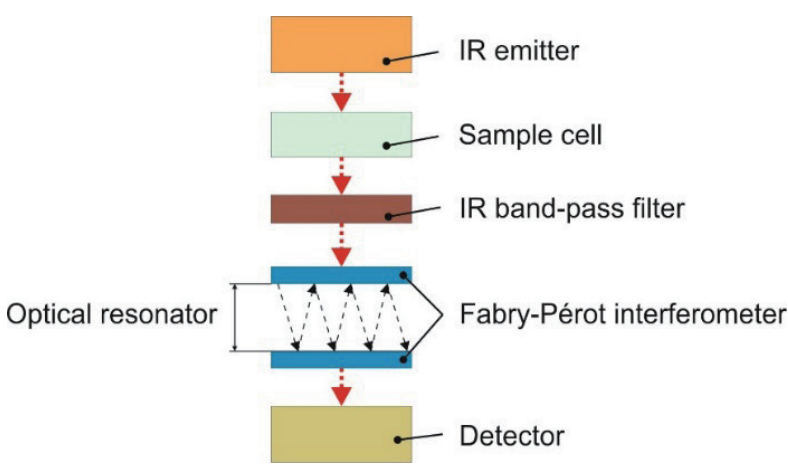

(b)

Fig. 4. (Color online) (a) Measured transmission spectrum of the FPI for different tuning voltages and (b) schematic drawing of a spectrometer setup with FPI. 
Packaging, and MITNETZ STROM have developed the ASTROSE ${ }^{\circledR}$ system over the last few years.

\section{1 $\quad$ ASTROSE $^{\circledR}$ sensor network}

The ASTROSE ${ }^{\circledR}$ sensor network (Fig. 5) consists of numerous sensor nodes measuring the inclination, temperature, and current load of the spans of a high-voltage power line. A single sensor node (Fig. 6) consists of a microcontroller, a real-time clock, an electronic energy management system, and a $2.4 \mathrm{GHz}$ wireless transceiver. To determine the inclination and therefore the temperature of the conductor, a two-axis precession capacitive MEMS sensor based on the Air Gap Insulated Microstructures (AIM) technology has been developed. This high-resolution and temperature-stable sensor with a special application specific integrated circuit (ASIC) supplies a resolution of $0.01^{\circ}$. Thus, a change in the length of the conductors of 5 $\mathrm{mm}$ and a change in the sag of $22 \mathrm{~mm}$ can be resolved.

Equipped with two antennas and an additional antenna filter, the components are housed in a cylindrical and conducting hull with a slot for easy mounting on the power line (Fig. 7). On an operating power line, the sensor node requires no battery. As a power source, the electrostatic fringing field of the high-voltage power line is used. ${ }^{(10,11)}$ The collected data are passed wirelessly along the sensor chain and transmitted periodically to a base station (Fig. 5). In Refs. 12 and 13, the system is described in detail.

\subsection{Field results}

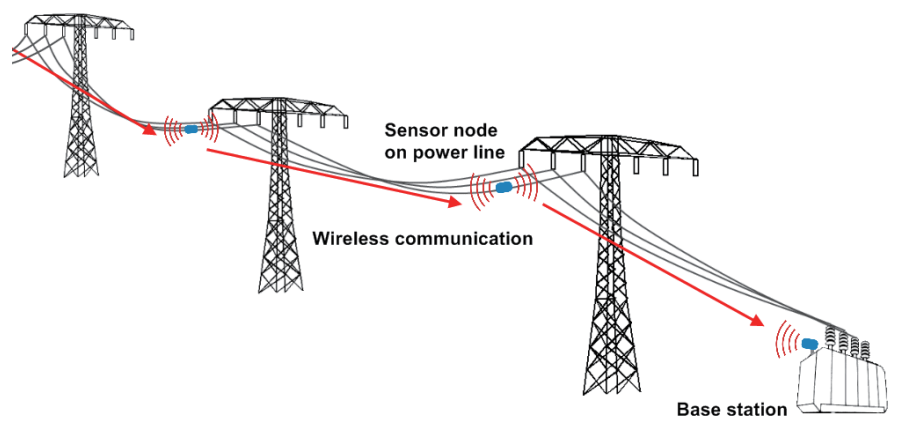

Fig. 5. (Color online) ASTROSE® sensor network.

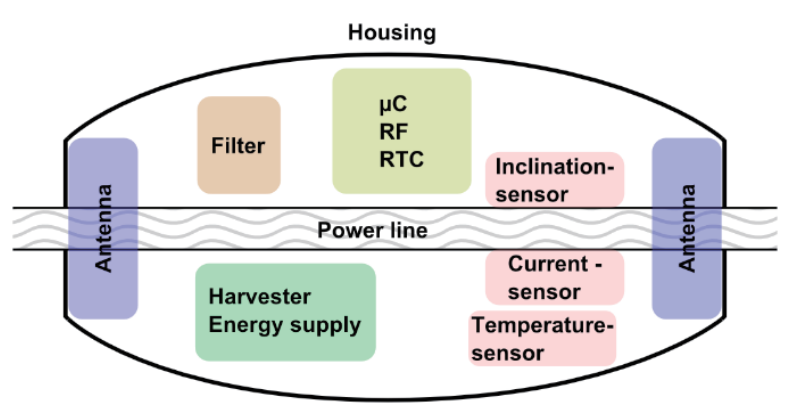

Fig. 6. (Color online) Block diagram sensor node.

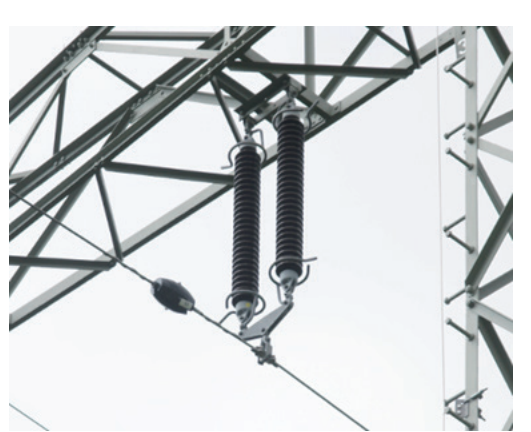

Fig. 7. Sensor node on a power line. 


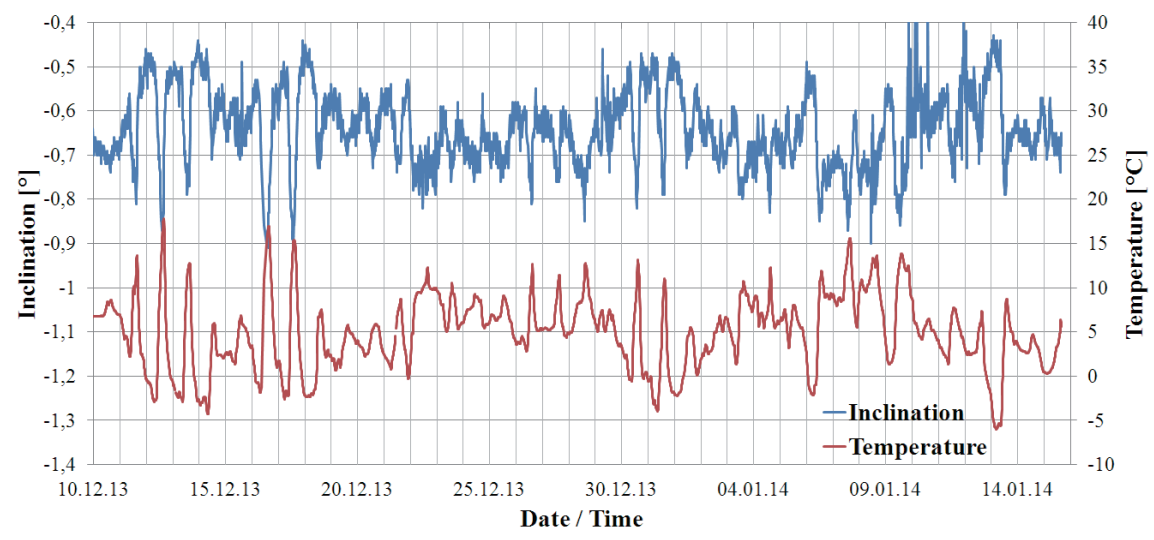

Fig. 8. (Color online) Inclination and temperature variation during field test.

An extensive test run of the sensor network was started in September 2012 and is still ongoing. The recorded data (inclination, temperature, and current) were analyzed and compared with weather data of the test area and current load values of the power line. Figure 8 shows the typical correlation of temperature and inclination. Higher temperature leads to a higher negative inclination, and it is seen that the inclination sensor resolves the fluctuation much faster than the temperature sensor that has a certain measurement delay owing to its thermal capacitance and limited thermal conductance between the temperature sensor and the power cable.

\section{Smart Systems for Production}

Current industrial trends in mechanical engineering and plant manufacturing address condition monitoring mainly to minimize system failures. The implementation of sensors, electronics for signal conditioning, wireless signal transmission (necessary due to rotating parts), and self-sustaining power supply allows an autonomous and efficient operation of such systems for various applications. Therefore, nanotechnologies play an important role.

Nanotechnology is often mentioned as one of the key enabling technologies in this century. It allows the development of new products in nearly all fields of our daily life, e.g. electronics, medical technology, and the automotive industry. Owing to their manifold properties and appearing effects, nanomaterials, such as nanoparticles, nanorods, nanowires, and nanotubes, are a very productive source for the development of innovative microsystems. Moreover, nanocomposites and nanostructures provide completely new opportunities for the realization of sensor and actuator systems in microsystem technology. Processing from liquid dispersions via cost-saving technologies, such as printing, allows for new sensor or actuator designs, even on flexible and curved surfaces. Consequently, sensor arrays can be manufactured easily on films, which in turn can act as semifinished products for further processing, e.g., for integration in 
fiber-reinforced plastic (FRP) materials.

FRPs offer excellent mechanical properties at low weight. That is why such lightweight structures are increasingly used in aerospace, automotive engineering, shipbuilding, and in the field of renewable energies. However, in contrast to metals, lightweight materials are sensitive to mechanical impacts, which may lead to damage (microcracks, inner delamination) within the material. Additionally, penetrating water can alter the mechanical properties of FRPs markedly. Loss of stiffness, and finally, complete failure of the structure might be the consequence. To reduce the mentioned negative effects, boost the performance of lightweight materials, and increase safety, it is reasonable to monitor such structures continuously with respect to mechanical overloads and water uptake. Both issues can be handled with appropriate nanocomposites, as will be outlined next.

For the monitoring of mechanical impacts on lightweight structures, we have recently presented a novel concept of a self-sufficient and flexible thin-film sensor. ${ }^{(14,15)}$ Our approach is to use a piezoelectric material in combination with quantum dots (QDs) for the detection and visualization of mechanical impacts by influencing QD photoluminescence (PL) properties by charge carriers. ${ }^{(16,17)}$ A mechanical load, acting on the layer system, causes the generation of charge carriers in the piezoelectric transducer, which are subsequently injected into QDs. Charge injection in QDs causes a reduction in photoluminescence intensity, which has the potential to indicate the position and strength of the acting load. Hence, the status of the filmbased sensor can be determined by illumination with ultraviolet (UV) light. In the case of an unloaded sensor, the initial PL intensity is emitted, while in case of an applied load, the PL intensity is reduced locally, leading to an optical contrast. Since the charges are stored within the QDs, also the PL state remains visible for a certain time, which allows the visualization of load events occured. QD sensors on polyethylene terephthalate (PET) film as well as piezo elements have been integrated in glass-FRPs via the resin infusion process (Fig. 9).

Composites have also been used to manufacture humidity sensors for monitoring the water uptake of FRP components. Instead of quantum dots, ceramic nano- and microparticles in a

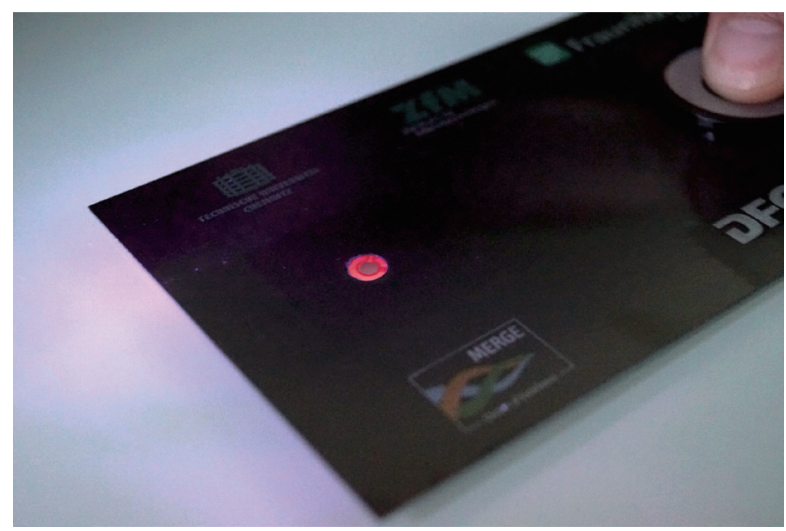

Fig. 9. (Color online) QD load sensor in glass-FRPs. 
polymer matrix serve as water-sensitive dielectrics in a capacitive humidity sensor setup. ${ }^{(18,19)}$ Since the dielectric permittivity of water is much larger than that of the ceramic-polymer composite, the adsorbed water changes the dielectric permittivity markedly, and hence the capacitance of the setup. Thus, it is not surprising that a more than $200 \%$ capacitance change could be detected in the range between 10 and $80 \%$ relative humidity. For the integration of the sensors in lightweight materials, the following route was used: first, preparation of sensors on film via screen or flexographic printing followed by the attachment of the sensor on glassfiber-reinforced polyamide prepreg (GF-PA6) and contacting via soutache embroidery; finally, thermal consolidation of several prepreg layers to a laminate with incorporated humidity sensor. The water uptake of the laminate during water storage is clearly detected by the incorporated sensor. Thereby, the sensor signal is in good agreement with water diffusion properties of the material. From the sensor characteristics, it can be specified that a mean water uptake of approx. 0.5 wt.\% can be determined reliably.

The examples of load detection/impact visualization and the monitoring of absorbed water show that nano- and microcomposites are ideal materials for material-integrated sensing and actuating. Although the sensors are demonstrated with special FRPs, the preparation and integration technologies are not limited to this type of material. Substrate as well as the active materials can be modified in a wide range, so that nanocomposites in particular become allround building blocks for future microsystems.

\section{Conclusions}

Global demands for highly integrated smart systems are set to dramatically increase in the years to come. From the application viewpoint, smart production, industry 4.0, smart home, smart city, smart energy, smart mobility, smart health, and smart society require systems with increased functionality, operating safety, security and reliability.

By looking at future research challenges for sensing devices, different tendencies can be observed. As the systems will combine more and more functionalities, the power consumption of each system plays an important role. For that reason, low-power-consumption sensors and actuators, low-power electronics as well as wake-up receivers are under development. Moreover, more and more smart materials will be developed: for example, nanotechnologies and nanomaterials will be used because of their advantageous properties.

However the Internet of Things requires not only the further development of reliable lowcost sensing and actuating systems but also the further development of data processing and cloud computing, which will lead to smarter systems. Many software aspects have to be taken into account, from data security to the handling of big data, for the knowledge database.

\section{References}

1 SRA2017, EPoSS: https://www.smart-systems-integration.org/public/documents/publications/EPoSS SRA2017.

2 T. Gessner and T. Köhler: Proc. Smart Systems Integration SSI 2012 (2012) Paper 0.

3 C. Gnoth and S. Johnson: Geburtsh. Frauenheilk. 74 (2014) 661.

4 B. Gehr: MMW-Fortschritte der Medizin 159 (2017) 58.

5 M. Ebermann, N. Neumann, K. Hiller, M. Seifert, M. Meinig, and S. Kurth: Proc. SPIE 8977 (2014) 89770T. 
6 M. Noro, K. Suzuki, N. Kishi, H. Hara, T. Watanabe, and H. Iwaoko: Proc. IEEE MEMS 2003 Conf. (2003) 319-322.

7 P. A. Stupar, R. L. Borwick, J. F. DeNatale, P. H. Kobrin, and W. J. Gunning: Proc. IEEE Transducers 2009 Conf. (2009) 1357-1360.

8 N. Neumann, M. Ebermann, S. Kurth, and K. Hiller: J. Micro/Nanolith. MEMS MOEMS 7 (2008) 02100401-021004-9.

9 M. Meinig, M, Ebermann, N. Neumann, S. Kurth, K. Hiller, and T. Gessner: Proc. IEEE Transducers Conf. (2011) $2538-2541$

10 H. Zangl, T. Bretterklieber, and G. Brasseur: IEEE Trans. Instr. Meas. 58 (2009) 1789.

11 T. Keutel, X. Zhao, and O. Kanoun: Proc. SENSOR+TEST Conference 2009 (2009).

12 S. Voigt, M. Nowack, A. Bertz, S. Leidich, M. Meinig, S. Kurth, T. Gessner, T. Keutel, X. Zhao, O. Kanoun, S. Lissek, H. During, B. Rusek, C. Brockmann, V. Grosser, M. Braunschweig, and R. Krumm: Proc. Mikrosystemtechnik Kongress 2011 (2011) paper 88.

13 S. Voigt, J. Wolfrum, M. Pfeiffer, T. Keutel, C. Brockmann, V. Großer, S. Lissek, H. During, B. Rusek, M. Braunschweig, S. Kurth, and T. Gessner: Proc. VDE-Kongress Smart Grid-Intelligente Energieversorgung der Zukunft (2012).

14 M. Moebius, J. Martin, M. Hartwig, R. R. Bauman, and T. Otto: Proc. Smart Systems Integration 2017 (2017) 423-426.

15 M. Moebius, J. Martin, E. A. Poppitz, P. Ueberfuhr, G. Tofighi, O. D. Gordan, V. M. Dzhagan, H. Lang, R. R. Baumann, D. R. T. Zahn, T. Otto, T. Gessner, and L. Kroll: Proc. Smart System Integration 2014 (2014) $543-546$

16 M. Moebius, J. Martin, M. Hartwig, R. R. Baumann, T. Otto, and T. Gessner: AIP Adv. 6 (2016) 085309.

17 M. Moebius, X. Ma, J. Martin, M. F. Doty, T. Otto, and T. Gessner: Proc. SPIE 9370 (2015).

18 T. Seider, J. Martin, A.Boeddicker, J. Rühling, D. Wett, D. Nestler, G. Wagner, A. C. Huebler, T. Otto, and T. Gessner: Mat. Sci. Forum 825-826 (2015) 579-585.

19 J. Martin, K. Shetty, N. Reimann, S. Neukirchner, U. Fügmann, H. Illing-Günther, D. Nestler, A. C. Hübler, K. Nendel, L. Kroll, and T. Otto: Proc. 3rd Int. MERGE Technologies Conf. 2017 (2017) 121.

\section{About the Authors}

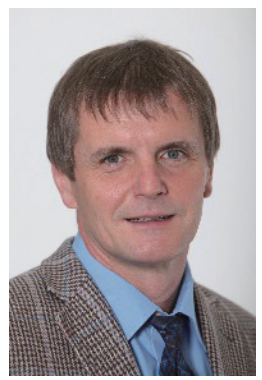

Thomas Otto studied electrical engineering and completed his doctoral thesis for Dr--Ing. at TH Karl-Marx-Stadt in 1987. He earned his postdoctoral lecturing qualification for Dr.-Ing. habil. in the field of electrical engineering/ information technology at Chemnitz University of Technology in 1996. From 1998 he was group leader/ head of department at Fraunhofer IZM, Department of Multi Device Integration. From 2008 he was deputy head of the Fraunhofer-Institute for Electronic Nano Systems ENAS (7/2008-12/2010 Fraunhofer Research Institution for Electronic Nano Systems). Since May 2016 he is the Acting Director of the Fraunhofer Institute for Electronic Nano Systems ENAS. Moreover, since October 2016 he is the Chair for Microtechnology at Chemnitz University of Technology and the Director of the Center for Microtechnologies of the Chemnitz University of Technology.

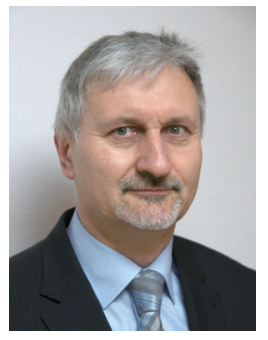

Steffen Kurth received his Dr.-Ing. degree in MEMS modeling and test from Chemnitz University of Technology in 1995. He is active in the design, application and test of MEMS, MOEMS and RF-MEMS, and he leads the department of Micro Device Integration at the Fraunhofer Research Institute for Electronic Nano Systems (ENAS) in Chemnitz, Germany. MEMS scanners, tunable IR filter, MEMS switches and sensor applications in smart systems for infrastructure monitoring and precision farming are the main topics of his research currently. 


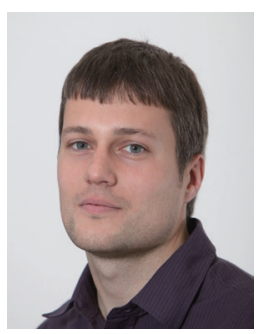

Sven Voigt received his Dipl.-Ing. degree in Electrical Engineering from Chemnitz University of Technology, Germany, in 2007. Thereafter at the university, he worked on printed organic transistors, RF MEMS/NEMS devices and sensor systems. In 2013 he joined the Fraunhofer Institute for Electronic Nano Systems, where he continues to focus on RF MEMS/NEMS devices and sensor systems.

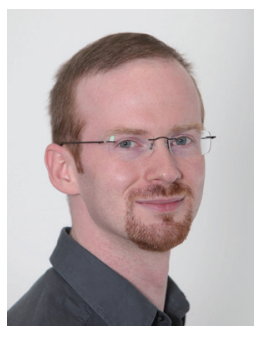

Andreas Morschhauser received his diploma in Microsystem Technology from the University of Applied Sciences, Zwickau (Germany) in 2006. From 2006 to 2011 he worked on polymer metallization and integration of novel materials into microfluidic systems at the Center for Microtechnologies (TU Chemnitz, Germany). Since 2011, he has been working on microfluidic systems of decentralized analysis and especially on microfluidic sample preparation at Fraunhofer ENAS.

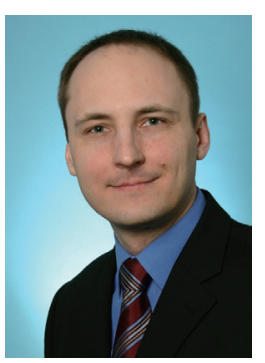

Marco Meinig received his Diploma in Electrical Engineering from Chemnitz University of Technology, Germany, in 2007. Thereafter, he was with the Center for Microtechnologies of Chemnitz University of Technology, and since 2008 he has been with the Fraunhofer Institute for Electronic Nano Systems, Chemnitz. His major research interests include silicon-based optical microsystems, microspectrometers, hyperspectral imaging and novel methods for the characterization of MEMS and MOEMS.

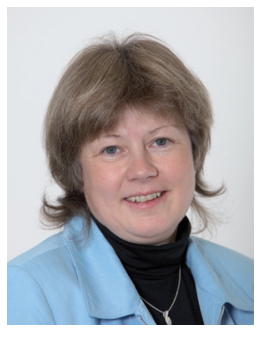

Karla Hiller finished her diploma study of Electrotechnical Engineering at TU Dresden in 1988. After that, she started to work at Chemnitz University of Technology in the field of microsystem technology with focus on wafer bonding technology, and she finished her Ph.D. in 1994. Thereafter, she developed MEMS technologies for several MEMS applications, such as sensors for acceleration, vibration, angular rate, pressure, and optical filters and micromirrors. In 2004, she obtained her habilitation in the field of microtechnology. Since 2008, she has been Deputy Director of the Center for Microtechnologies and since 2011 she has worked as professor at the TU Chemnitz.

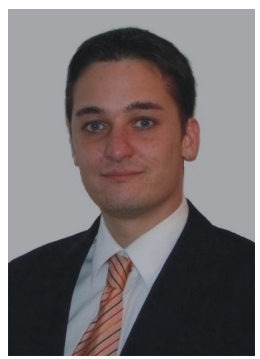

Martin Moebius completed his diploma thesis in February 2011 at Technische Universität Chemnitz and works since March 2011 at the Center for Microtechnologies (ZfM). Since the beginning of 2013 he is involved in research activities in Clusters of Excellence "MERGE Technologies for Multifunctional Lightweight Structures". 


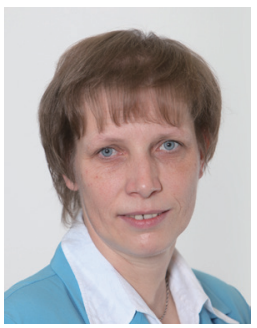

Martina Vogel studied physics at the University of Technology Chemnitz, Germany. She obtained her Ph.D. from the same university in 1994. From 1996 to 2001, she worked as project manager in GPP Chemnitz mbH. From 2001 to 2006, she was responsible for the quality assurance of memory products at ZMD. In 2006, she joined the Center for Microtechnologies of Technische University Chemnitz. Since 2009, she has been with Fraunhofer ENAS and works as advisor to the institute management and manager marketing/PR. Since 2015, she has been working additionally as strategy coordinator of the institute. 